\title{
Bioassay Guided Fractionation of an Anti-Methicillin-Resistant Staphylococcus aureus Flavonoid From Bromus inermis Leyss Inflorescences
}

\author{
Atousa Aliahmadi ${ }^{1,} ;$ Fateme Mirzajani $^{2,3}$; Alireza Ghassempour ${ }^{4} ;$ Ali Sonboli $^{1}$ \\ ${ }^{1}$ Department of Biology, Medicinal Plants and Drug Research Institute, Shahid Beheshti University, Tehran, IR Iran \\ ${ }^{2}$ Department of Biotechnology, Faculty of Renewable Energies and New Technologies Engineering, Shahid Beheshti University, Tehran, IR Iran \\ ${ }^{3}$ Department of Nanobiotechnology, Protein Research Institute, Shahid Beheshti University, Tehran, IR Iran \\ ${ }_{4}^{4}$ Department of Phytochemistry, Medicinal Plants and Drug Research Institute, Shahid Beheshti University, Tehran, IR Iran \\ ${ }^{*}$ Corresponding author: Atousa Aliahmadi, Department of Biology, Medicinal Plants and Drug Research Institute, Shahid Beheshti University, Tehran, IR Iran. Tel/Fax:+98-02122431783,
} E-mail:a_aliahmadi@sbu.ac.ir

Received: July 23, 2013; Revised: September 22, 2013; Accepted: October 5, 2013

\begin{abstract}
Background: Plants are considered as promising sources of new antibacterial agents as well as bioassay guided fractionation.
Objectives: In the present work, the antibacterial properties, especially against methicillin-resistant Staphylococcus aureus (MRSA), of Bromus inermis inflorescence was studied, using the bioassay guided fractionation as well as the bio-autographic method.

Materialsand Methods:The plant organic extract was prepared via maceration in methanol, followed by the fractionation using n-hexane. The extracts were subjected for minimum inhibitory concentrations (MICs) against some human pathogenic bacteria via standard broth micro-dilution assay. Thereafter, a bio-autographical method was applied using the high performance thin layer chromatography(HPTLC) coupled with agar overlay assays for the primary characterization and identification of bioactive substance (s).

Results: Through the bioassay guided fractionation method, the greatest antibacterial activities were related to the n-hexane extract. It was also revealed that the effective anti-MRSA agent of the assessed plant was a relatively polar substance with an MIC value of about $8 \mu \mathrm{g}$ $\mathrm{mL}$ against the tested MRSA strain (in comparison with the MIC value of $32 \mu \mathrm{g} / \mathrm{mL}$ for chloramphenicol).

Conclusions: As a result of the full range UV-Vis scanning of the responsible band in the HPTLC experiments (200-700 nm), the flavonoid was the most imaginable natural compound.
\end{abstract}

Keywords: Chemical Fractionation; Methicillin-Resistant Staphylococcus aureus, Flavonoid, Anti-bacterial Agents

\section{Background}

The emergence of multidrug-resistant human pathogens is considered as a major concern worldwide. Bacterial pathogens become resistant to the current therapies and transfer the resistance elements to each other via sophisticated systems (1). Plants have somehow known systemic or local defense mechanisms. For this necessity, they produce some family of secondary metabolites, reactive oxygen species, and antimicrobial peptides against bacterial pathogens. Production of these antimicrobials may be constitutive or inducible in different environmental conditions. Moreover, some plant antimicrobials may be reserved or secreted by organisms (2-4).

Weed can be a great source of antibacterial compounds regarding its ability to survive in varied difficult growth conditions $(2,4,5)$, including contamination with bacterial pathogens. According to the antibacterial properties of some Iranian weeds, Bromus inermis Leyss extract exhibits a remarkable inhibitory effect on some assessed bacterial strains. This plant is a potent weed and belongs to the Poaceae family (6).

\section{Objectives}

In the present study, the bioassay-guided fractionation method was used to monitor the inflorescence and potential antibacterial effects of the B. inermis Leyss extract.

\section{Materials and Methods}

\subsection{Microbial Strains}

In this study, microbial strains including Staphylococcus aureus ATCC 25923, methicillin-resistant S. aureus (MRSA) and the vancomycin-resistant strain of Enterococcus faecium (kindly dedicated by Prof. M. M. Feizabadi), E. faecalis ATCC 29212, Escherichia coli ATCC 25922, Salmonella enteritidis, Pseudomonas aeruginosa PTCC 1430, P. aeruginosa JH10 and P. aeruginosa JH5 (kindly dedicated by Prof. Jose L. Martínez) were studied.

\subsection{Plant and Chemicals Preparation}

The inflorescence of B. inermis was obtained from Pakan Bazr Co., Isfahan, Iran. The organic solvents were purchased from Caledon Lab (Ontario, Canada). The reagents and the high performance thin layer chromatography

Copyright (C) 2014,Ahvaz Jundishapur University of Medical Sciences; Published by Kowsar. This is an open-access article distributed under the terms of the Creative Commons Attribution License, which permits unrestricted use, distribution, and reproduction in any medium, provided the original work is properly cited. 
(HPTLC) silica plates were provided by Merck Co., (KGaA, Darmstadt, Germany).

\subsection{Plant Extraction}

Selected uniform plants were milled and soaked with methanol in a dark glass under constant stirring for 48 hours at room temperature. Thereafter, the mixture was filtered through a filter paper and the filtrate was dried at $40^{\circ} \mathrm{C}$ using a rotary evaporator instrument (Heidolph Co., UK). Of the remaining, $1 \mathrm{mg}$ was dissolved in $5 \mathrm{~mL}$ of water. The extract was further extracted using equal volumes of n-hexane solvent for removing oily and nonpolar ingredients the process was repeated twice more. The organic extract was dried under a reduced pressure at $40^{\circ} \mathrm{C}$ and was kept at $4^{\circ} \mathrm{C}$. In a parallel experiment, extraction was carried out using n-hexane, following methanol to extract the polar compounds.

\subsection{Antimicrobial Assay}

A stock of methanol as well as the n-hexane extract powder were dissolved in dimethyl sulfoxide (DMSO) and stored at $4^{\circ} \mathrm{C}$. Antimicrobial susceptibility test was performed using the broth micro-dilution method for determination of the minimum inhibition concentration (MIC) and the minimum bactericidal concentration (MBC), as recommended by the Clinical and Laboratory Standard Institute of the US (CLSI) (7), with some modifications. Briefly, an 18-hour culture of each test microorganism was used for the susceptibility tests. A serial dilution of each extract (from $64 \mathrm{mg} / \mathrm{mL}$ to $0.006 \mathrm{mg} / \mathrm{mL}$ ) was prepared using sterile, Muller Hinton broth (MHB) (Merck \& Co., Inc. Germany), containing 0.5\% Tween 80 . Tween 80 was used as a co-solvent for better solvation of the plant extract in the MHB medium.

Microorganisms were added to each well to final concentrations of $5 \times 10^{5} \mathrm{CFU} / \mathrm{mL}$ of bacteria. The inoculated 96-well plates were incubated for 20 hours at $37^{\circ} \mathrm{C}$ for all the bacteria, but at $35^{\circ} \mathrm{C}$ for the MRSA strain. The results were recorded as the lowest concentration that could inhibit visible growth of microorganisms (MICs). The plant extracts were chromatic, so in some cases it was necessary to use a growth indicator instead of checking the wells turbidity. Therefore, the Resazurin (Sigma-Aldrich, US.) reagent was used as a growth indicator, as illustrated by Sarker et al. (8). MBCs were determined by sub-culturing $100 \mu \mathrm{L}$ of each negative well on nutrient agar plates. The results were recorded after 24 hours of incubation at appropriate temperatures, as the lowest concentrations that could kill $99.99 \%$ of the initial microorganisms was determined during the quality control step, as recommended by CLSI (5). All the experiments were performed in triplicates.

\subsection{High Performance Thin Layer Chromatography}

HPTLC was carried out using CAMAG HPTLC instrument (Muttenz, Switzerland). A 1-mg/mL clear solution of $\mathrm{n}$ hexane extract was dissolved in an n-hexane and ethyl acetate $(1: 1, \mathrm{v} / \mathrm{v})$ solvent mixture and was spotted onto aluminum silica gel plates PF254 under $\mathrm{N}_{2}$, using Linomat 5 , under $0.2 \mu \mathrm{L} / \mathrm{sec}$ speed. Each 6 -mm band contained about $30 \mu \mathrm{g}$ of the extract. A variety of mobile phases were used for achieving the highest separation resolution. All the plates were scanned in the range of $180-800 \mathrm{~nm}$, using CAMAG TLC scanner III (9).

\subsection{Bio-Autography Experiments}

The developed TLC plates with the highest resolutions were assessed against an important antibiotic-resistant bacterium, a clinical strains of MRSA. In brief, a $50^{\circ} \mathrm{C}$ Mueller Hinton agar (MHA) medium, containing $10^{7} \mathrm{CFU} /$ $\mathrm{mL}$ of bacteria, was poured into silica plates together with the resolved bands and then incubated for 18 hours at $37^{\circ} \mathrm{C}(10)$. An MHA medium supplemented with $2 \%$ $\mathrm{NaCl}$ was also used. The effective and ineffective bands were chosen through comparison of the normal scanned plates (under $254 \mathrm{~nm}, 366 \mathrm{~nm}$ and visible light) with the bio-autographic plates. Next, the effective and ineffective bands were cut and subjected against the bacteria.

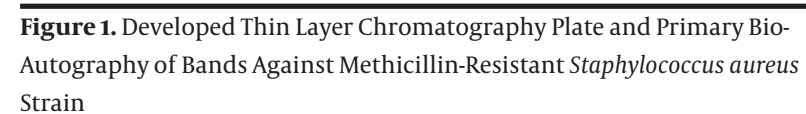
Strain
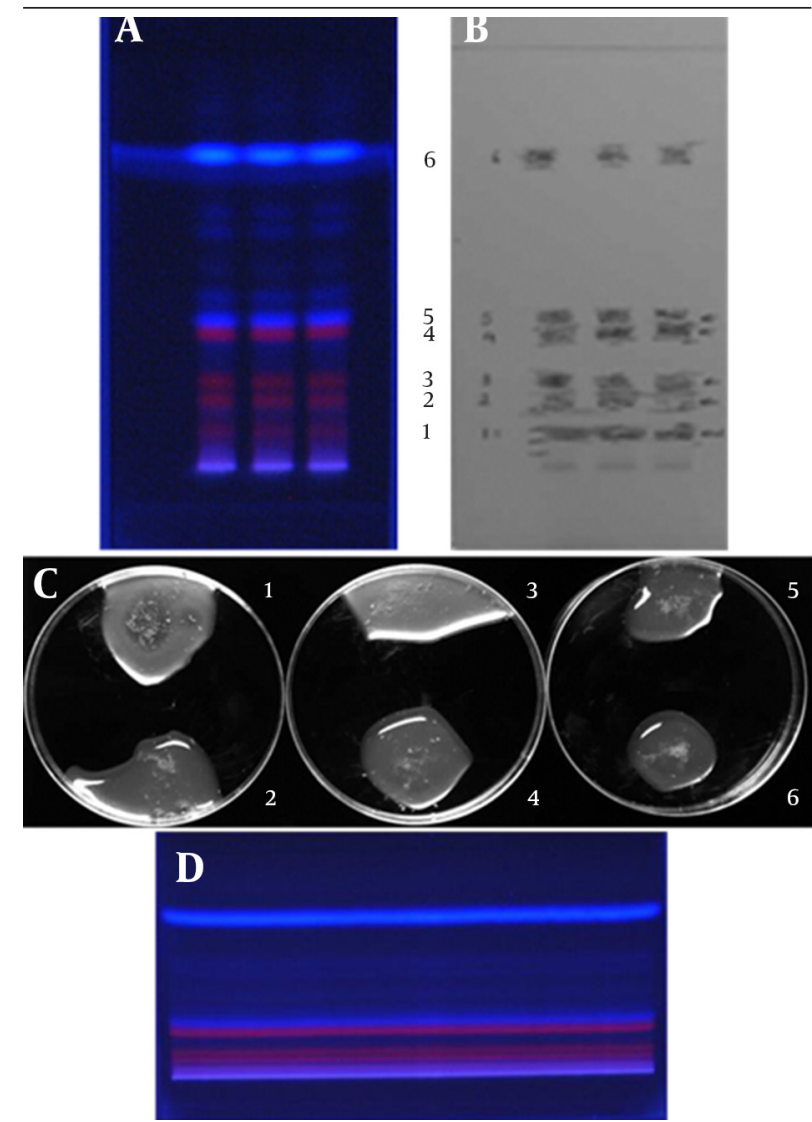

A: developed plate under $366 \mathrm{~nm}$ UV. B: developed plate after cutting of the resolved bands. C:1-6, different bands after addition of MHA containing $10^{7} \mathrm{CFU} / \mathrm{mL}$ MRSA strain. As shown in Figure $1 \mathrm{C}$, there is a clear zone of inhibition around the silica powder containing band 1. D: prepared aluminum sheet PF254 after resolving the bands under $366 \mathrm{~nm}$ light. 
3.7. Semi-Purification of Effective Substance (s) Using Preparative Thin Layer Chromatography

A total of $1500 \mu \mathrm{g}$ n-hexane extract solution was loaded into aluminum silica gel plates PF254 (Figure 1D) and the bands were resolved, as mentioned in section 3-5. The effective band (band 1 in Figure $1 \mathrm{~A}$ ) was scraped from the developed sheet, immersed in methanol, and dissolved using 30 seconds of sonication. MIC of the filtrate was determined against the MRSA strain.

\subsection{Total Flavonoid Content Determination}

Total flavonoid content of the semi-purified sample was determined by the aluminum chloride colorimetric method and the total flavonoid content was expressed as mg quercetin equivalents (QE) (11).

\section{Results and Discussion}

The primary screening of 15 different methanol and nhexane plant extracts against 12 different microorganisms (a collection of bacteria and yeast, data are not shown), indicated the inflorescence of $B$. inermis Leyss. Those results demonstrated a remarkable inhibition of Gram-positive bacteria, but a relatively slight activity against Gramnegative ones. The antibacterial effects of plant extracts are presented in Table 1. It is apparent that not only the overall antibacterial effects of the n-hexane extract were better than the methanol extract, but also it had better inhibitory effects against Gram-positives rather than Gramnegative bacteria. This extract inhibited the growth of $S$. aureus and E. faecalis at concentrations of 0.06 and 0.125 $\mathrm{mg} / \mathrm{mL}$, respectively. However, the best results against the Gram-negative strains were achieved by E. coli and S. enteritidis strains ( 1 and $2 \mathrm{mg} / \mathrm{mL}$, respectively).

In the HPTLC experiments, the best resolution of bands was gained using n-hexane: ethyl acetate $(4: 1 \mathrm{v} / \mathrm{v})$ developing solvent system (the chromatography was repeated twice) (Figure $1 \mathrm{~A}$ ). According to the Bio-autography experiments, there were clear zones of inhibition around the bottom of the developed silica plate, which for the MRSA strain, they were repeated using MHA supplemented by $2 \% \mathrm{NaCl}$ (Figure 2). As shown in Figure 1 (A, B and C), for identification of the bands containing effective antibacterial substances, different bands were cut from the developed plates and assessed against the MRSA strain. The results revealed that band 1 contained anti-MRSA substance (s). Full range scanning of this band showed that it may be a member of flavonoids. As shown in Figure 3, the maximum absorbance of the substance was in the range of $250-254 \mathrm{~nm}$, which seems to be the maximum absorbance of flavonoids (12).

The semi-purified antibacterial substance was subjected to more study of its total flavonoid content and antibacterial activity. Its total flavonoid content was about 86 $\mathrm{mg} \mathrm{QE} / \mathrm{g}$. The anti-bacterial effects as well as the total flavonoid content results may confirm the flavonoid identity of the chosen substance (s). While the MRSA strain could be inhibited by $32 \mu \mathrm{g} / \mathrm{mL}$ of chloramphenicol, MIC of semi-purified substance was about $8 \mu \mathrm{g} / \mathrm{mL}$ against the tested bacterium.

Table 1. Antibacterial Activities of Methanol and n-Hexane Extracts of Bromus Inermis a,b

\begin{tabular}{lcc}
\hline & Methanol Extract & n-Hexane Extract \\
\hline S. aureus & & \\
MIC & 4 & 0.06 \\
\hline MBC & 16 & 0.5 \\
\hline MRSA strain & & \\
\hline MIC & 16 & 0.125 \\
\hline MBC & 32 & 2 \\
\hline VRE strain & & \\
\hline MIC & 32 & 0.5 \\
\hline MBC & 32 & 8 \\
\hline E. faecium & & \\
\hline MIC & 16 & 42 \\
\hline MBC & 0.5 & 42 \\
\hline
\end{tabular}

\section{E. faecalis}

$\begin{array}{ccc}\text { MIC } & 8 & 16 \\ \text { MBC } & 0.125 & 0.5 \\ \text { E. coli } & & \\ \text { MIC } & 16 & 1 \\ \text { MBC } & 16 & 4\end{array}$

S. enteritidis

\begin{tabular}{|ccc|} 
MIC & 18 & 2 \\
MBC & 16 & 4 \\
\hline P. aeruginosa $\mathrm{JH5}^{\mathrm{C}}$ & & \\
MIC & 64 & 16 \\
MBC & $>64$ & 64 \\
\hline
\end{tabular}

P. aeruginosa $\mathrm{JH}_{10}^{\mathrm{d}}$

\begin{tabular}{lcc} 
MIC & 32 & 8 \\
MBC & $>64$ & 64 \\
\hline P. aeruginosa $^{\mathrm{e}}$ & & \\
MIC & 16 & 4 \\
MBC & 64 & 16 \\
\hline
\end{tabular}

a Abbreviations: MBC; minimum bactericidal concentration; MIC, minimum inhibitory concentration; MRSA, methicillin-resistant Staphylococcus aureus; VRE, vancomycin resistant Entreococcus faecium

$\mathrm{b}$ MIC and MBC are in $\mathrm{mg} / \mathrm{mL}$.

${ }^{\text {c }}$ P. aeruginosa JH5: Multidrug Resistant Efflux Pump Bearing Strain.

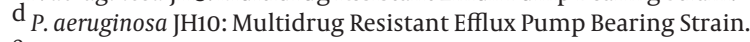

e P. aeruginosa PTCC1340. 
Figure 2. Bio-Autography of n-Hexane Extract Resolved in Silica Plate Against Methicillin-Resistant Staphylococcus aureus

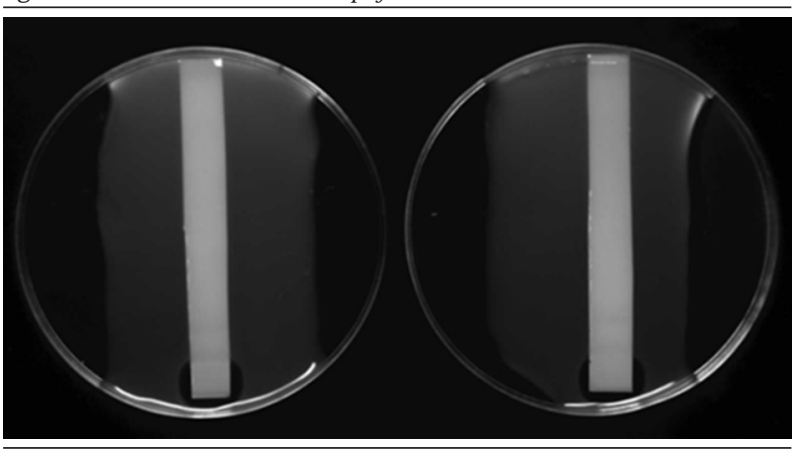

Right: MHA with 2\% NaCl; left: MHA medium without NaCl.

Substance 3 on all Tracks

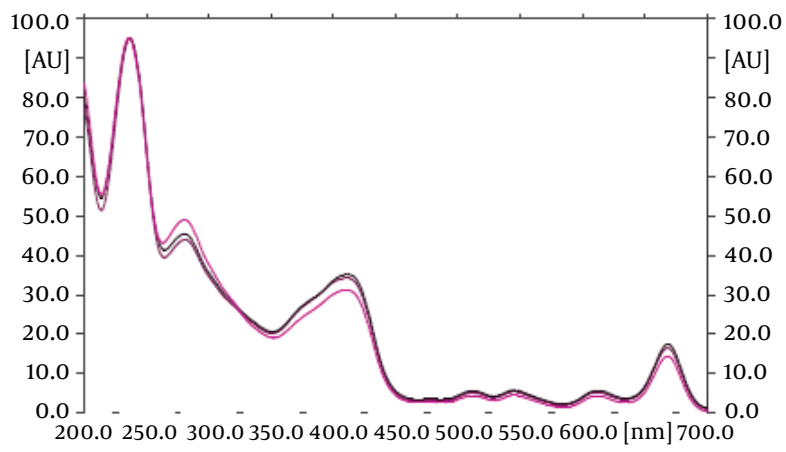

\begin{tabular}{ccll}
$\mathrm{T}$ & $\mathrm{Rf}$ & Substance & Max.@ \\
\hline 2 & $0.21 \mathrm{RF}$ & Substance 3 & $237 \mathrm{~nm}$ \\
3 & $0.21 \mathrm{RF}$ & Substance 3 & $237 \mathrm{~nm}$ \\
4 & $0.21 \mathrm{RF}$ & Substance 3 & $237 \mathrm{~nm}$ \\
\hline
\end{tabular}

Figure 3. Scan of the Effective Band in 200-700 nm in Three Repeats

Weeds can be the promising sources of finding new and more effective antibacterial substances because of their characteristics related to their dominancy in severe growth and living conditions. To the best of our knowledge, it was the first report on such great antibacterial activity regarding the inflorescence of $B$. inermis weed plant. It can hypothesize that further investigations with HPLC fractionation and subsequent nuclear magnetic resonance (NMR) analysis could reveal the identity of the effective substances in our continuous research. Such antibacterial substances can also be considered as the lead compounds for semi-synthesis of new effective antibiotics.

\section{Acknowledgements}

The authors would like to thank Dr. M. Moridi for his kind guides in HPTLC experiments. Shahid Beheshti University, G.C. (600/2146) provided the financial support of this study.

\section{Authors' Contributions}

Atousa Aliahmadi contributed to preparation of the plant extracts, performing the antibacterial tests and drafting the manuscript. Fateme Mirzajani conducted the HPTLC experiments. Alireza Ghassempour supervised the HPTLC experiments. Ali Sonboli contributed to the identification of plant samples as a plant systematic professional.

\section{Funding/Support}

Shahid Beheshti University, G.C. (600/2146) provided the financial support of this study. The funding organizations were academic institutions and had no role in the design and conduct of the study, collection, management and analysis of the data, or preparation, review and approval of the manuscript.

\section{References}

1. Wenzel RP, Edmond MB. The impact of hospital-acquired bloodstream infections. Emerg Infect Dis. 2001;7(2):174-7.

2. Maroti G, Kereszt A, Kondorosi E, Mergaert P. Natural roles of antimicrobial peptides in microbes, plants and animals. Res Microbiol. 2011;162(4):363-74.

3. Cowan MM. Plant products as antimicrobial agents. Clin Microbiol Rev. 1999;12(4):564-82.

4. Thevissen K, Kristensen HH, Thomma BP, Cammue BP, Francois IE. Therapeutic potential of antifungal plant and insect defensins. Drug Discov Today. 2007;12(21-22):966-71.

5. Odintsova TI, Rogozhin EA, Baranov Y, Musolyamov A, Yalpani N Egorov TA, et al. Seed defensins of barnyard grass Echinochloa crusgalli (L.) Beauv. Biochimie. 2008;90(11-12):1667-73.

6. Watson L, Dallwitz MJ. The grass genera of the world: descriptions, illustrations, identification, and information retrieval; including synonyms, morphology, anatomy, physiology, phytochemistry, cytology, classification, pathogens, world and local distribution, and references. 1992. Available from: http://delta-intkey.com.

7. Jorgensen JH, Turnidge JD. Susceptibility test methods: Dilution and disk diffusion methods. In: Murray PR, Baron EJ, Jorgensen JH, Landry MR, Pfaller MA editors. Manual of Clinical Microbiology. 9 ed. Washington DC: ASM PRESS; 2007. pp. 1152-72.

8. Sarker SD, Nahar L, Kumarasamy Y. Microtitre plate-based antibacterial assay incorporating resazurin as an indicator of cell growth, and its application in the in vitro antibacterial screening of phytochemicals. Methods. 2007;42(4):321-4.

9. Waksmundzka-Hajnos M, Sherma J, Kowalska T. Thin Layer Chromatography in Phytochemistry.: Taylor \& Francis; 2008.

10. Mirhosseini M, Nahvi I, Emtiazi G, Tavassoli M. Characterisation of anti-Listeria monocytogenesbacteriocins fromEnterococcus faeciumstrains isolated from dairy products.Int J Dairy Tech. 2010;63(1):55-61.

11. Huck CW, Buchmeiser MR, Bonn GK. Fast analysis of flavonoids in plant extracts by liquid chromatography-ultraviolet absorbance detection on poly(carboxylic acid)-coated silica and electrospray ionization tandem mass spectrometric detection. J Chromatogr A. 2002;943(1):33-8.

12. Zhishen J, Mengcheng T, Jianming $\mathrm{W}$. The determination of flavonoid contents in mulberry and their scavenging effects on superoxide radicals. Food Chem. 1999;64(4):555-9. 